\title{
Analytical Algorithm for Systems of Neutral Delay Differential Equations
}

\author{
Aminu Barde ${ }^{1,2}$, Normah Maan ${ }^{*}$ \\ ${ }^{1}$ Department of Mathematical Sciences, University Technology Malaysia, Skudai, Malaysia \\ ${ }^{2}$ Department of Mathematical Sciences, Abubakar Tafawa Balewa University, Bauchi, Nigeria \\ Email: bardealamin@yahoo.com, *normahmaan@utm.my
}

How to cite this paper: Barde, A. and Maan, N. (2019) Analytical Algorithm for Systems of Neutral Delay Differential Equations. Applied Mathematics, 10, 753-768. https://doi.org/10.4236/am.2019.109054

Received: August 27, 2019

Accepted: September 22, 2019

Published: September 25, 2019

Copyright $\odot 2019$ by author(s) and Scientific Research Publishing Inc. This work is licensed under the Creative Commons Attribution International License (CC BY 4.0).

http://creativecommons.org/licenses/by/4.0/

\section{c) (i) Open Access}

\begin{abstract}
Delay differential equations (DDEs), as well as neutral delay differential equations (NDDEs), are often used as a fundamental tool to model problems arising from various areas of sciences and engineering. However, NDDEs particularly the systems of these equations are special transcendental in nature; it has therefore, become a challenging task or times almost impossible to obtain a convergent approximate analytical solution of such equation. Therefore, this study introduced an analytical method to obtain solution of linear and nonlinear systems of NDDEs. The proposed technique is a combination of Homotopy analysis method (HAM) and natural transform method, and the He's polynomial is modified to compute the series of nonlinear terms. The presented technique gives solution in a series form which converges to the exact solution or approximate solution. The convergence analysis and the maximum estimated error of the approach are also given. Some illustrative examples are given, and comparison for the accuracy of the results obtained is made with the existing ones as well as the exact solutions. The results reveal the reliability and efficiency of the method in solving systems of NDDEs and can also be used in various types of linear and nonlinear problems.
\end{abstract}

\section{Keywords}

Homotopy Analysis Method, Natural Transform, He's Polynomial and Neutral Delay Differential Equations

\section{Introduction}

Ordinary differential equations (ODEs) are usually used as a fundamental tool in modelling the problems of the real world. However, in most cases, the mathematical formulation of real-life problems needs to consider both the present and past states of the system behaviour. Different from (ODEs), DDE is a type of 
differential equation in which the derivative of the unknown function at a certain time is given in terms of the values of the function at previous time [1]. Hence, more reliable models of real problems arising from various field of studies such as; biology, population dynamics, chemistry, and physics, control theory to mention but few are now model using DDEs as well as NDDEs [2] [3] [4] [5] [6]. Recently series of methods have been developed to find an approximate analytical solution to different types of DDEs [7]-[12]. However, most of these methods have experienced a series of challenges in finding a convergent approximate analytical solution of NDDEs in particular system of such equations. So, scientist and engineers adopt the use of numerical methods as the best approach to approximate the solutions. Therefore, more analytical approaches are highly needed for solving these equations.

This work aims to develop an analytical technique for solving linear and nonlinear systems of NDDEs from the combination of HAM and Natural transform. The proposed technique improved on the work of Rebenda and Smarda [12] by introducing the concept of NDDE into Natural transform. In addition, the various derivatives for both proportional and constants delay of NDDEs were successfully generated using the Natural transform. This work is also an extension of Efficient Analytical Approach for Nonlinear System of Retarded Delay Differential equations. This approach was developed by Barde and Maan [13] and solutions to different types of nonlinear systems of retarded DDEs were obtained in a series form which converges to exact or approximate solution.

Thus, based on the results of this pervious works, this research focuses to develop a new analytical technique that modifies Efficient Analytical Approach for Nonlinear System of Retarded Delay Differential Equations with aims to obtain approximate analytical solution for both linear and nonlinear system of NDDEs with proportional and constant delays. Using the introduced technique, the He's polynomial is adjusted in order to ease the computational difficulties of nonlinear terms of such equations. Furthermore, the convergence analysis and the maximum estimated error of the technique are also investigated.

Therefore, in this work we were able to develop a new generating function in Equation (29) that provides a convergent analytical solution to various types of linear and nonlinear system of NDDEs in a series form using few numbers of computational terms and minimal error as compared with the previous techniques. Thus, different from some of the existing methods the presented technique provides solution to different form of linear and nonlinear NDDEs without any linearization, perturbation or unnecessary assumptions. In Section 4, some illustrative examples are presented in order to show the reliability and efficiency of our algorithms over the reference methods.

\section{Methods}

The idea of this work is come up with analytical approach from the combination of natural transform and HAM for solving systems of linear and nonlinear NDDEs. The He's polynomial is modified to compute the series of nonlinear 
terms of both proportional and constants delays.

HAM is a powerful technique introduced by Lio [14] for solving different types of linear and nonlinear problems. Details on theory and application of HAM can be found in [1] [15] [16] [17] [18].

In recent years natural transform is considered as an active topic in research due to its vast application in solving different type of differential and integral equations [19] [20] [21] [22]. This transform was derived from the renowned Fourier integral which converged to either Laplace or Sumudu transforms depending on the values of the transform variables. The basic concepts of natural transform for further use in this research are rendered below.

Definition 2.1 [23] Let $t \in(-\infty, \infty)$, then the natural transform of the function $v(t)$ is defined by.

$$
\mathbf{N}^{+}[v(t)]=V(s, u)=\int_{-\infty}^{\infty} \mathrm{e}^{-s t} v(u t) \mathrm{d} t ; \quad s, u \in(0, \infty] .
$$

where $\mathbf{N}^{+}$denotes as natural transform and $s, u$ are transforming variables.

Equation (1) can be simplified as [23]

$$
\begin{aligned}
\mathbf{N}^{+}[v(t)] & =V(s, u)=\int_{-\infty}^{\infty} \mathrm{e}^{-s t} v(u t) \mathrm{d} t ; \quad s, u \in(0, \infty] \\
& =\int_{-\infty}^{0} \mathrm{e}^{-s t} v(u t) \mathrm{d} t ; s, u \in(-\infty, 0)+\int_{0}^{\infty} \mathrm{e}^{-s t} v(u t) \mathrm{d} t ; s, u \in(0, \infty) \\
& =\mathbf{N}^{-}[v(t)]+\mathbf{N}^{+}[v(t)] \\
& =\mathbf{N}[v(t) H(-t)]+\mathbf{N}[v(t) H(t)] \\
& =V^{-}(s, u)+V^{+}(s, u)
\end{aligned}
$$

where, $H($.$) is the Heaviside function. Assume the function v(t) H(t)$ is defined on $\mathbf{R}^{+}$and for $t \in \mathbf{R}$ then its natural transform can be define over the set

$$
A=\left\{v(t): \exists M, \tau_{1}, \tau_{2}>0,|v(t)|<M \mathrm{e}^{\frac{|t|}{\tau_{j}}}, t \in(-1)^{j} \times[0, \infty), j \in \mathbf{Z}^{+}\right\}
$$

as in the given integral:

$$
\mathbf{N}^{+}[v(t)]=V^{+}(s, u)=\int_{0}^{\infty} \mathrm{e}^{-s t} v(u t) \mathrm{d} t ; \quad s, u \in(0, \infty] .
$$

Theorem 2.1 [24] The generalised natural transform of the function $v(t)$ is given as

$$
\mathbf{N}^{+}[v(t)]=V^{+}(s, u)=\sum_{n=0}^{\infty} \frac{n ! a_{n} u^{n}}{s^{n+1}} .
$$

Property 2.1 [23] Let a be a non-zero constant and $v($ at $) \in A$ then,

$$
\mathbf{N}^{+}[v(a t)]=\frac{1}{a} V\left(\frac{s}{a}, u\right) .
$$

Theorem 2.2 [20] If $\mathrm{H}_{\tau}$ is the Heaviside function and for any real number $\tau \geq 0$ we defined 


$$
H_{\tau}(t)= \begin{cases}1, & \text { for } t \geq \tau \\ 0 & \text { for } t<\tau\end{cases}
$$

Then the natural transform of the shifted function $v(t-\tau)=v(t-\tau) H_{\tau}(t)$ is given by

$$
\mathbf{N}^{+}\left[v(t-\tau) H_{\tau}(t)\right]=\mathrm{e}^{\frac{-s \tau}{u}} \mathbf{N}^{+}[v(t)]
$$

Theorem 2.3 [24] Let $v^{(n)}(t)$ be the nth derivatives of the function $v(t)$ then its natural transform is given by

$$
\mathbf{N}^{+}\left[v^{n}(t)\right]=V_{n}^{+}(s, u)=\frac{s^{n}}{u^{n}} V(s, u)-\sum_{k=1}^{n} \frac{s^{n-k}}{u^{(n-k)+1}} v^{k-1}(0) .
$$

\section{Corollary 2.1}

Let $v_{i}^{(n)}(a t)$ be the $n t h$ derivatives of the functions $v_{i}(a t)$ with respect to $t$, $(i=1,2, \cdots, N)$ and suppose that $\mathbf{N}^{+}\left[v_{i}(a t)\right]=V_{i}^{+}(a s, u)$ then we define the following

$$
\mathbf{N}^{+}\left[v_{i}^{n}(a t)\right]=V_{n, i}^{+}(a s, u)=\frac{s^{n}}{(a u)^{n}} V_{i}^{+}(a s, u)-\sum_{k=1}^{n} \frac{s^{n-k}}{a u^{(n-k)+1}} v_{i}^{(k-1)}(0)
$$

\section{Proof}

Using an induction method, for $n=1$ and $n=2$ we respectively obtained the natural transform of first and second derivatives of $v_{i}(a t)$ that is

$$
\begin{aligned}
& \mathbf{N}^{+}\left[v_{i}^{\prime}(a t)\right]=V_{1, i}^{+}(a s, u)=\frac{s}{a u} V_{i}^{+}(a s, u)-\frac{v_{i}(0)}{a u} \\
& \mathbf{N}^{+}\left[v_{i}^{\prime \prime}(a t)\right]=V_{2, i}^{+}(a s, u)=\frac{s^{2} V_{i}^{+}(a s, u)-s v_{i}(0)}{(a u)^{2}}-\frac{v_{i}^{\prime}(0)}{a u}
\end{aligned}
$$

Now suppose the result holds for $n$, then we have to show its also true for $n+1$. Now from Equation (9) we have

$$
\begin{aligned}
\mathbf{N}^{+}\left[v_{i}^{(n+1)}(a t)\right] & =\mathbf{N}^{+}\left[\left(v_{i}^{(n)}(a t)\right)^{\prime}\right]=V_{n+1, i}^{+}(a s, u)=\frac{s}{a u} V_{n, i}^{+}(a s, u)-\frac{v_{i}^{(n)}(0)}{a u} \\
& =\frac{s}{a u}\left[\frac{s^{n}}{(a u)^{n}} V_{i}^{+}(a s, u)-\sum_{k=1}^{n} \frac{s^{n-k}}{(a u)^{(n-k)+1}} v_{i}^{(k-1)}(0)\right]-\frac{v_{i}^{(n)}(0)}{a u} \\
& =\frac{s^{n+1}}{(a u)^{n+1}} V_{i}^{+}(a s, u)-\sum_{k=1}^{n+1} \frac{s^{(n-k)+1}}{(a u)^{(n-k)+2}} v_{i}^{(k-1)}(0)
\end{aligned}
$$

which is true for $n+1$ and hence the result.

\section{Corollary 2.2}

Suppose $v_{i}^{(n)}(t-\tau)$ are the $n t h$ derivatives of the shifted functions $v_{i}(t-\tau)$ with respect to $t$, then their natural Transforms can be define as

$$
\begin{aligned}
\mathbf{N}^{+}\left[v_{i}^{n}(t-\tau)\right] & =\mathrm{e}^{\left(-\frac{s \tau}{u}\right)} V_{n, i}^{+}(s, u) \\
& =\frac{s^{n}}{u^{n}} \mathrm{e}^{\left(-\frac{s \tau}{u}\right)} V_{i}^{+}(s, u)-\sum_{k=1}^{n} \frac{s^{n-k}}{(u)^{(n-k)+1}}\left[\lim _{t \rightarrow 0} v_{i}^{(k-1)}(t-\tau)\right]
\end{aligned}
$$




\section{Proof}

Also by induction, for $n=1$ and $n=2$ we respectively have the natural transform of first and second derivatives of $v_{i}(t-\tau)$, that is

$$
\begin{aligned}
& \mathbf{N}^{+}\left[v_{i}^{\prime}(t-\tau)\right]=\frac{s}{u} \mathrm{e}^{\frac{-s \tau}{u}} V_{i}^{+}(s, u)-\frac{1}{u} \lim _{t \rightarrow 0} v_{i}(t-\tau) \\
& \mathbf{N}^{+}\left[v_{i}^{\prime \prime}(t-\tau)\right]=\frac{s^{2} \mathrm{e}^{\frac{-s \tau}{u}} V_{i}^{+}(s, u)-s \lim _{t \rightarrow 0} v_{i}(t-\tau)}{u^{2}}-\frac{\lim _{t \rightarrow 0} v_{i}^{\prime}(t-\tau)}{u}
\end{aligned}
$$

Now assume Equation (11) is true for $n$ and we have to show for $n+1$. From Equation (12) we have

$$
\begin{aligned}
& \mathbf{N}^{+}\left[v_{i}^{(n+1)}(t-\tau)\right]=\mathbf{N}^{+}\left[\left(v_{i}^{n}(t-\tau)\right)^{\prime}\right]=\frac{s}{u} \mathbf{N}^{+}\left[v_{i}^{(n)}(t-\tau)\right]-\frac{\lim _{t \rightarrow 0} v_{i}^{n}(t-\tau)}{u} \\
& =\frac{s}{u}\left[\frac{s^{n}}{u^{n}} \mathrm{e}^{\frac{-s \tau}{u}} V_{i}^{+}(s, u)-\sum_{k=1}^{n} \frac{s^{n-k}}{u^{(n-k)+1}}\left[\lim _{t \rightarrow 0} v_{i}^{(k-1)}(t-\tau)\right]\right]-\frac{\lim _{t \rightarrow 0} v_{i}^{(n)}(t-\tau)}{u} \\
& =\frac{s^{n}}{u^{n}} \mathrm{e}^{\frac{-s \tau}{u}} V_{i}^{+}(s, u)-\sum_{k=1}^{n+1} \frac{s^{n-k+1}}{u^{(n-k)+2}}\left[\lim _{t \rightarrow 0} v_{i}^{(k-1)}(t-\tau)\right]
\end{aligned}
$$

which is true for $n+1$ and hence the proof.

Another important point to note is that, in both the concept of HAM and natural transform there is no direct approach for the computation of nonlinear terms of the system of NDDEs for both Proportional and constant delays. Therefore, in this research the He's polynomial will be adjusted for the series calculation of these nonlinear terms.

\section{Analysis of the Result}

Consider the following $n$-order system of NDDEs

$$
\begin{aligned}
& {\left[v_{i}(t)+v_{i}(\alpha(t))\right]^{(n)}} \\
& =F_{i}\left[t, v_{\gamma}^{(p)}(t), v_{\gamma}^{(p)}\left(\alpha_{i, j}(t)\right)\right], t \in[0, d], i=1, \cdots, N, J=1, \cdots, M
\end{aligned}
$$

where

$$
\begin{aligned}
& v_{\gamma}^{(p)}(t)=\left(v_{1}^{(p)}, v_{2}^{(p)}, \cdots, v_{N}^{(p)}\right), \\
& v_{\gamma}^{p}\left(\alpha_{i, j}(t)\right)=\left(v_{1}^{(p)}\left(\alpha_{i, j}(t)\right), v_{2}^{(k)}\left(\alpha_{i, j}(t)\right), \cdots, v_{N}^{(p)}\left(\alpha_{i, j}(t)\right)\right)
\end{aligned}
$$

for $p=0,1,2, \cdots, n-1$ and $\alpha_{i, j}(t)$ are the functions of delay terms such that $\alpha(t)=\max \left[\alpha_{i, j}(t)\right]$

with the given initial conditions

$$
v_{i}^{(p)}(0)=v_{i, 0}^{(p)}, \quad v_{i}(t)=\psi_{i}(t), \quad t<0
$$

Now for simplicity we rewrite Equation (14) in the following form

$$
L_{i}\left(v_{i}+v_{i}(\alpha)\right)+R_{i}\left(v_{\gamma}\right)+F\left(v_{\gamma}\right)=g_{i}(t)
$$

Subject to a given initial conditions. The $v_{\gamma}$ is defined as $N$-dimentional vector of the form $v_{\gamma}=\left[v_{1}(t), v_{2}(t), \cdots, v_{N}(t)\right]$. The linear terms are decom- 
posed into bounded linear operators $L_{i}+R_{i}$ (That is there are some positive numbers $\alpha_{i, 1}, \alpha_{i, 2}$ such that $\left\{\left\|L_{i}\left(v_{\gamma}\right)\right\| \leq \alpha_{i, 1}\left\|v_{\gamma}\right\|,\left\|R_{i}\left(v_{\gamma}\right)\right\| \leq \alpha_{i, 2}\left\|v_{\gamma}\right\|\right)$ with $L_{i}$ as the highest order and $R_{i}$ as remaining of the linear operators, and $F_{i}$ are continuous functions satisfy the Lipschitz condition with Lipschitz constants $\left.\mu_{i} \in[0, d] \quad\left(\left|f_{i}(v)-f_{i}(u)\right| \leq \mu_{i}|v-u|, \forall t \in[0, d]\right\}\right)$ represent the non-linear terms.

Take the natural transform of both sides of Equation (16) to obtain:

$$
\mathbf{N}^{+}\left[L_{i}\left(v_{i}+v_{i}\left(\alpha_{i}\right)\right)\right]+\mathbf{N}^{+}\left[R_{i}\left(v_{\gamma}\right)\right]+\mathbf{N}^{+}\left[F_{i}\left(v_{\gamma}\right)\right]=\mathbf{N}^{+}\left[g_{i}(t)\right]
$$

Note: This research considered two forms of delay functions $\alpha_{i, j}(t)$ as follows:

Case I: $\alpha_{i, j}(t)=a_{i, j} t$, where $a_{i, j} \in(0,1)$ (proportional delay).

Case II: $\alpha_{i, j}(t)=t-\tau_{i, j}$, where $\tau_{i, j}>0$ are real constants (constant delay) Therefore, by substituting the given initial condition into Equation (17), and simplify using the differential properties of natural transform we respectivily obtained the following for the two types of delay as defined in Case I and Case II.

$$
\begin{aligned}
& \mathbf{N}^{+}\left[v_{i}(t)+\frac{1}{a_{i}^{(n+1)}} v\left(a_{i} t\right)\right]-\sum_{k=1}^{n}\left(1+\frac{1}{a_{i}^{(n+1-k)}}\right) \frac{u^{n-k}}{s^{k}} v_{i}^{k-1}(0) \\
& +\frac{u^{n}}{s^{n}} \mathbf{N}^{+}\left[R_{i}\left(v_{\gamma}\right)+F_{i}\left(v_{\gamma}\right)-g_{i}(t)\right]=0 \\
& \mathbf{N}^{+}\left[v_{i}(t)+\mathrm{e}^{\frac{-s \tau_{i}}{u}} v_{i}(t)\right]-\sum_{k=1}^{n} \frac{u^{n-k}}{s^{k}}\left[v_{i}^{k-1}(0)+\lim _{t \rightarrow 0} v_{i}^{k-1}\left(t-\tau_{i}\right)\right] \\
& +\frac{u^{n}}{s^{n}} \mathbf{N}^{+}\left[R_{i}\left(v_{\gamma}\right)+F_{i}\left(v_{\gamma}\right)-g_{i}(t)\right]=0
\end{aligned}
$$

where $a_{i}=\max \left[a_{i, j}\right]$ and $\tau_{i}=\max \left[\tau_{i, j}\right]$

Now from Equation (18) we can define the following nonlinear operators

$$
\begin{aligned}
N_{i}\left[\phi_{i}(t ; q)\right]= & \mathbf{N}^{+}\left[\phi_{i}(t ; q)+\frac{1}{a_{i}^{(n+1)}} \phi_{i}\left(a_{i} t ; q\right)\right]-\sum_{k=1}^{n}\left(1+\frac{1}{a_{i}^{(n+1-k)}}\right) \frac{u^{n-k}}{s^{k}} \phi_{i}^{k-1}(9) \\
& +\frac{u^{n}}{s^{n}} \mathbf{N}^{+}\left[R_{i}\left(\phi_{\gamma}(t ; q)\right)+F_{i}\left(\phi_{\gamma}(t ; q)\right)-g_{i}(t)\right] \\
N_{i}\left[\phi_{i}(t ; q)\right]= & \mathbf{N}^{+}\left[\phi_{i}(t ; q)+\mathrm{e}^{\frac{-s \tau_{i}}{u}} \phi_{i}(t ; q)\right]-\sum_{k=1}^{n} \frac{u^{n-k}}{s^{k}}\left[\phi_{i}^{k-1}(0)+\lim _{t \rightarrow 0} \phi_{i}^{k-1}\left(t ; q-\tau_{i}\right)\right] \\
& +\frac{u^{n}}{s^{n}} \mathbf{N}^{+}\left[R_{i}\left(\phi_{\gamma}(t ; q)\right)+F_{i}\left(\phi_{\gamma}(t ; q)\right)-g_{i}(t)\right]
\end{aligned}
$$

where $q \in[0,1]$ is an embedding parameter, $\phi_{i}(t ; q)$ are functions of variables $t$ and $q$.

So, by means of HAM we can construct the following Homotopy Equations as

$$
(1-q) \mathbf{N}^{+}\left[\phi_{i}(t ; q)-v_{i, 0}(t)\right]=h_{i} q H_{i}(t) N_{i}\left[\phi_{\gamma}(t ; q)\right]
$$

where $\mathbf{N}^{+}$denotes as natural transform, $v_{i, 0}(t)$ are initial approximations of $v_{i}(t)$ and $h_{i}, H(t)$ are non-zero auxiliary parameters and auxiliary fuctions respectively. 
Now, from Equation (20) as $q=0$ and $q=1$ we respectively obtained the following equation.

$$
\begin{aligned}
& \phi_{i}(t, 0)=v_{i, 0}(t) \\
& \phi_{i}(t, 1)=v_{i}(t)
\end{aligned}
$$

Thus, as $q$ increases from 0 to 1 , the solutions $\phi_{i}(t, q)$ vary from the initial approximations $v_{i, 0}(t)$ to the exact solutions $v_{i}(t)$. In topology, this type of variation is called deformation and Equation (20) is called zero-order deformation equation.

Therefore, the Taylor series expansion of $\phi_{i}(t, q)$ with respect to $q$ can be obtained as

$$
\phi_{i}(t, q)=\phi_{i}(t, 0)+\sum_{m=1}^{\infty} v_{i, m}(t) q^{m}
$$

where

$$
v_{i m}(t)=\left.\frac{1}{m !} \frac{\partial^{m} \phi_{i}(t ; q)}{\partial q^{m}}\right|_{q=0}
$$

Suppose that the initial approximations $v_{i, 0}(t)$, auxiliary parameters $h_{i}$ and the auxiliary function $H_{i}(t)$ are properly chosen so that the series in Equation (22) converges at $q=1$, that is

$$
\phi_{i}(t, 1)=v_{i, 0}(t)+\sum_{m=1}^{\infty} v_{i, m}(t)
$$

Define vectors

$$
\boldsymbol{v}_{i, n}(t)=\left[v_{i, 0}(t), v_{i, 1}(t), \cdots, v_{i, n}(t)\right]
$$

By differentiate Equation (20) $m$ times with respect to $q$ and setting $q=0$ and finally divided by $m$ ! we obtain the so called $m t h$-order deformation equation as

$$
\mathbf{N}^{+}\left[v_{i, m}(t)-\chi_{m} v_{i, m-1}(t)\right]=h_{i} H_{i}(t) R_{y_{i} m}\left[v_{i, m-1}(t)\right]
$$

where

$$
R_{y_{i}, m}\left[\boldsymbol{v}_{i, m-1}(t)\right]=\left.\frac{1}{(m-1) !} \frac{\partial^{m-1} N_{i}\left[\phi_{i}(t, q)\right]}{\partial q^{m-1}}\right|_{q=0}
$$

and

$$
\chi_{m}= \begin{cases}0, & m \leq 1 \\ 1, & m>1\end{cases}
$$

By taking the inverse natural transform on both sides of Equation (25) we obtained

$$
v_{i, m}(t)=\chi_{m} v_{i, m-1}(t)+h_{i} \mathbf{N}^{-}\left[H_{i}(t) R_{y_{i} m}\left[\boldsymbol{v}_{i, m-1}(t)\right]\right]
$$

Therefore, $v_{i, m}(t)$ for $m \geq 1$ can be easily obtained from Equation (27), at $M$ th order we have 


$$
v_{i}(t)=\sum_{m=0}^{M} v_{i, m}(t)
$$

Hence, as $M \rightarrow \infty$ the following recursive relations of Equations (14) and (15) for the two type of delay as defined respectively in Case I and Case II are obtained

$$
\begin{aligned}
v_{i, m}(t)= & \left(\chi_{m}+h_{i}\right) v_{i, m-1}(t)+h_{i} \frac{1}{a_{i}^{n}} v_{i, m-1}\left(a_{i} t\right) \\
& -h_{i}\left(1-\chi_{m}\right) \mathbf{N}^{-} \sum_{k=1}^{n}\left(1+\frac{1}{a_{i}^{n-k+1}}\right) \frac{u^{k-1}}{s^{k}} v_{i}^{k-1}(0) \\
& +h_{i} \mathbf{N}^{-}\left\{\frac{u^{n}}{s^{n}} \mathbf{N}^{+}\left[R_{i}\left(v_{\gamma, m-1}(t)\right)+H_{i, m-1}\left(v_{\gamma_{1}}, v_{\gamma_{2}}, \cdots, v_{\gamma_{N}}\right)-g_{i}(t)\right]\right\}, m \geq 1 \\
v_{i, m}(t)= & \left(\chi_{m}+h_{i}\right) v_{i, m-1}(t)+h_{i} v_{i, m-1}\left(t-\tau_{i}\right) \\
& -h_{i}\left(1-\chi_{m}\right) \mathbf{N}^{-} \sum_{k=1}^{n} \frac{u^{k-1}}{s^{k}}\left[v_{i}^{k-1}(0)+\lim _{t \rightarrow 0} v_{i}^{k-1}\left(t-\tau_{i}\right)\right] \\
& +h_{i} \mathbf{N}^{-}\left\{\frac{u^{n}}{s^{n}} \mathbf{N}^{+}\left[R_{i}\left(v_{\gamma, m-1}(t)\right)+H_{i, m-1}\left(v_{\gamma_{1}}, \cdots, v_{\gamma_{N}}\right)-g_{i}(t)\right]\right\}, m \geq 1
\end{aligned}
$$

Now, the nonlinear operators $F_{i}\left(v_{\gamma}\right)$ are expanded as series of modified He's polynomials $\left.H_{i, m-1}\left(v_{\gamma 1}, v_{\gamma 2}, \cdots, v_{\gamma n}\right)\right)$ define as

$$
H_{i, m}\left(v_{\gamma 1}, v_{\gamma 2}, \cdots, v_{\gamma N}\right)=\left.\frac{1}{m !} \frac{\partial^{m}}{\partial q^{m}} F_{i}\left(\sum_{p=0}^{m} q^{p} v_{\gamma, p}\right)\right|_{q=0}
$$

where $v_{\gamma, i}=\left(v_{i, 1}, v_{i, 2}, \cdots, v_{i, N}\right)$ and $v_{\gamma, p}=\left(v_{1, p}, v_{2, p}, \cdots, v_{N, p}\right)$.

The proof for Case II (constant delay) is of the same process with that of Case I.

Theorem 3.1 Assume $(C[D],\|\|$.$) is a Banach Space and let v_{i, m}(t)$ be defined in $(C[D],\|\|$.$) , where \left\|v_{i, m}\right\|$ are define in form of an operators, that is $v_{i, m}(t)=A_{i}\left(v_{i, m-1}(t)\right)$ such that $A_{i}(v)=-\mathbf{N}^{-} R_{y_{i}, m}\left[\vec{v}_{i, m-1}(t)\right]$ and

$$
\left\|A_{i}(v)-A_{i}(u)\right\| \leq \delta_{i}\|u-v\|, \quad \forall v, u \in C[D]
$$

where $\delta_{i}=\left(\alpha_{i, 1}+\alpha_{i, 2}+\mu_{i}\right) d$ for some $\delta_{i} \in(0,1)$. Then $A_{i}$ have unique fixed points in $C[D]$. Furthermore, the Homotopy series in Equation (23) converged uniquelly to the solutions $v_{i}(t)$ (their respective fixed points in $C[D]$ ) of Equations (14) and (15).

\section{Proof}

Let $[C(D),\|\cdot\|]$ be the Banach space of all continuous functions on $C(D)$, and from definitions of $L_{i}, R_{i}$ and $F_{i}$ in Equation (16) then we have to show that the $\left\{v_{i, m}\right\}$ are Cauchy sequences in $[C(D),\|\|$.$] . Now from Equation (19)$ we have

$$
\begin{aligned}
\left\|v_{i, m}-v_{i, p}\right\|= & \left\|-\mathbf{N}^{-}\left[R_{y_{i}, m}\left[\boldsymbol{v}_{i, m-1}(t)\right]-R_{y_{i}, p}\left[\boldsymbol{v}_{i, p-1}(t)\right]\right]\right\| \\
= & \| \mathbf{N}^{-}\left[\mathbf{N}^{+}\left[2\left(\phi_{i}(t ; q)+\frac{1}{a_{i}^{(n+1)}} \phi_{i}\left(a_{i} t ; q\right)\right)\right]\right. \\
& -\sum_{k=1}^{n}\left(1+\frac{1}{a_{i}^{(n+1-k)}}\right) \frac{u^{n-k}}{s^{k}}\left(\phi_{i, m}^{k-1}(0)-\phi_{i, p}^{k-1}(0)\right)
\end{aligned}
$$




$$
\begin{aligned}
& +\frac{u^{n}}{s^{n}} \mathbf{N}^{+}\left[R_{i}\left(\phi_{i, m-1}(t ; q)-\phi_{i, p-1}(t ; q)\right)\right. \\
& \left.\left.+F_{i}\left(\phi_{i, m-1}(t ; q)-\phi_{i, p-1}(t ; q)\right)-g_{i}(t)\right]\right] \| \\
\leq & \left\|\mathbf{N}^{-}\left\{\mathbf{N}^{+}\left[L_{i}\left(v_{i, m-1}-v_{i, p-1}\right)+R_{i}\left(v_{i, m-1}-v_{i, p-1}\right)+F_{i}\left(v_{i, m-1}-v_{i, p-1}\right)\right]\right\}\right\| \\
\leq & \left(\alpha_{i, 1}+\alpha_{i, 2}+\mu_{i}\right)\left\|v_{i, m-1}-v_{i, p-1}\right\| \leq \delta_{i}\left\|v_{i, m-1}-v_{i, p-1}\right\|
\end{aligned}
$$

For $m=p+1$, we have

$$
\left\|v_{i, p+1}-v_{i, p}\right\| \leq \delta_{i}\left\|v_{i, p}-v_{i, p-1}\right\| \leq \delta_{i}^{2}\left\|v_{i, p-1}-v_{i, p-2}\right\| \leq \cdots \leq \delta_{i}^{q}\left\|v_{i, 1}-v_{i, 0}\right\|
$$

Hence, for all $m, p \in N$ with $m \geq p$, by means of Equation (33) and using triangle inequality we seccessively obtained the following

$$
\begin{aligned}
\left\|v_{i, m}-v_{i, p}\right\| & \leq\left\|v_{i, m}-v_{i, m-1}\right\|+\left\|v_{i, m-1}-v_{i, m-2}\right\|+\cdots+\left\|v_{i, p+1}-v_{i, p}\right\| \\
& \leq \delta_{i}^{m-1}\left\|v_{i, 1}-v_{i, 0}\right\|+\delta_{i}^{m-2}\left\|v_{i, 1}-v_{i, 0}\right\|+\cdots+\delta_{i}^{p}\left\|v_{i, 1}-v_{i, 0}\right\| \\
& =\delta_{i}^{p}\left\|v_{i 1}-v_{i, 0}\right\| \sum_{k=0}^{m-n-1} \delta_{i}^{k} \leq \delta_{i}^{p}\left\|v_{i, 1}-v_{i, 0}\right\| \sum_{k=0}^{\infty} \delta_{i}^{k} \\
& =\delta_{i}^{p}\left\|v_{i 1}-v_{i, 0}\right\|\left(\frac{1}{1-\delta_{i}}\right) .
\end{aligned}
$$

Since $\delta_{i}<1$ then for arbitrary $\varepsilon_{i}$ we can find some large $\eta_{i} \in N$ such that

$$
\delta_{i}^{\eta_{i}}<\frac{\varepsilon_{i}\left(1-\delta_{i}\right)}{\left\|v_{i, 1}-v_{i, 0}\right\|}
$$

Therefore, choosing $\quad p, m>N$ then we obtain the following

$$
\left\|v_{i, m}-v_{i, p}\right\| \leq \delta_{i}^{p}\left\|v_{i, 1}-v_{i, 0}\right\|\left(\frac{1}{1-\delta_{i}}\right)<\frac{\varepsilon_{i}\left(1-\delta_{i}\right)}{\left\|v_{i, 1}-v_{i, 0}\right\|}\left\|v_{i, 1}-v_{i, 0}\right\|\left(\frac{1}{1-\delta_{i}}\right)=\varepsilon_{i}
$$

This shows that $\left\{v_{i, m}\right\}$ are Cauchy sequences in $C[D]$, and hence the sequences converged. And the Proof is now completed. The proof for Case II (constant delay) is of the same process with that of Case I.

To establish the proof for the uniqueness of these solutions, let $v_{i}(t)$ and $u_{i}(t)$ be two distinct solutions of Equations (14) and (15). According to Equation (16) we have

$$
v_{i}(t)+v_{i}(\alpha)=L_{i}^{-1}\left[g_{i}(t)-R_{i}\left(v_{\gamma}\right)-F_{i}\left(v_{\gamma}\right)\right]
$$

where $L_{i}^{-1}$ are the inverse operators defined by $\int_{0}^{t}(.) \mathrm{d} t$. Since $v_{i}(t)$ and $u_{i}(t)$ are distinct solutions of Equations (14) and (15), so from Equation (36) we obtain the following equation

$$
\begin{aligned}
& \left\|\left(v_{i}-u_{i}\right)+\left(v_{i, \alpha}-u_{i, \alpha}\right)\right\| \\
& \leq\left\|\left(v_{i}-u_{i}\right)\right\|=\left\|-\int_{0}^{t}\left[R_{i}\left(v_{i}-u_{i}\right)+F_{i}\left(v_{i}-u_{i}\right)\right] \mathrm{d} t\right\| \\
& \leq \int_{0}^{t}\left[\left\|R_{i}\left(v_{i}-u_{i}\right)\right\|+\left\|F_{i}\left(v_{i}\right)-F_{i}\left(u_{i}\right)\right\|\right] \mathrm{d} t \\
& \leq\left(\alpha_{2,1}\left\|v_{i}-u_{i}\right\|+\mu_{i}\left\|v_{i}-u_{i}\right\|\right) d \leq \delta_{i}\left\|v_{i}-u_{i}\right\|
\end{aligned}
$$


According to Equation (37) we have $\left(1-\delta_{i}\right)\left\|v_{i}-u_{i}\right\| \leq 0$ and since $\delta_{i} \in(0,1)$ then $\left\|v_{i}-u_{i}\right\| \leq 0$ implies that $v_{i}=u_{i}$ and hence the proof.

Theorem 3.2 Suppose the Homotopy series in Equation (23) converges to the solutions $v_{i}(t)$ of Equations (14) and (15) and let the approximations of $v_{i}(t)$ are given by the truncuted series $\sum_{m=0}^{M} v_{i, m}(t)$. Then the maximum absolute error is estimated to be

$$
\left\|v_{i}(t)-\sum_{m=0}^{M} v_{i, m}(t)\right\| \leq \frac{\delta_{i}^{n}}{1-\delta_{i}}\left\|v_{i, 0}\right\|
$$

\section{Proof}

According to Theorem 3.1 and Equation (34) we have

$$
\left\|v_{i, m}-v_{i, p}\right\| \leq \frac{\delta_{i}^{n}}{1-\delta_{i}}\left\|v_{i, 0}(t)\right\|
$$

As $m \rightarrow \infty$ then $v_{i, m} \rightarrow v_{i}(t)$, so we have

$$
\left\|v_{i}(t)-v_{i, p}\right\| \leq \frac{\delta_{i}^{n}}{1-\delta_{i}}\left\|v_{i, 0}(t)\right\|
$$

Since $\delta_{i} \in(0,1)$ then $1-\delta_{i} \leq 1$ and from Equation (39) we have

$$
\left\|v_{i}(t)-\sum_{m=0}^{M} v_{i, m}(t)\right\| \leq \frac{\delta_{i}^{n}}{1-\delta_{i}}\left\|v_{i, 0}(t)\right\|
$$

The Proof is now complete.

\section{Examples and Discussion}

The application of the proposed technique will be presented in this section. This involve solving some problems of linear and nonlinear systems of NDDEs with both proportional and constant delays.

Example 4.1 [10] First we seek for a solution of the following 2-dimensional linear system of NDDEs with constant delay

$$
\begin{gathered}
v_{1}^{\prime}(t)=v_{1}^{\prime}(t-1)+4 v_{2}(t), \quad 0 \leq t \leq 2 \\
v_{2}^{\prime}(t)=v_{1}(t)-v_{1}(t-1), \quad 0 \leq t \leq 2 \\
v_{1}(t)=\mathrm{e}^{-2 t}, \quad v_{2}(t)=\frac{1}{2}\left(\mathrm{e}^{-2(t-1)}-\mathrm{e}^{-2 t}\right), \quad t \in[-1,0] .
\end{gathered}
$$

Take the natural transform to both sides of Equation (41) and simplify further using Equation (11) to get

$$
\begin{aligned}
& \mathbf{N}^{+}\left[v_{1}(t)-\mathrm{e}^{-\frac{s}{u}} v_{1}(t)\right]-\frac{1}{s}\left[1-\mathrm{e}^{2}\right]-\frac{u}{s} \mathbf{N}^{+}\left[4 v_{2}(t)\right]=0 \\
& \mathbf{N}^{+}\left[v_{2}(t)\right]-\frac{1}{s}\left[\frac{1}{2}\left(\mathrm{e}^{2}-1\right)\right]+\frac{u}{s} \mathbf{N}^{+}\left[v_{1}(t-1)-v_{1}(t)\right]=0
\end{aligned}
$$

From Equation (42) define a non-linear operator

$$
\begin{aligned}
& N\left[\phi_{1}(t ; q)\right]=\mathbf{N}^{+}\left[\phi_{1}(t ; q)-\mathrm{e}^{-\frac{s}{u}} \phi_{1}(t ; q)\right]-\frac{1}{s}\left[1-\mathrm{e}^{2}\right]-\frac{u}{s} \mathbf{N}^{+}\left[4 \phi_{2}(t ; q)\right] \\
& N\left[\phi_{2}(t ; q)\right]=\mathbf{N}^{+}\left[\phi_{2}(t ; q)\right]-\frac{1}{s}\left[\frac{1}{2}\left(\mathrm{e}^{2}-1\right)\right]+\frac{u}{s} \mathbf{N}^{+}\left[\phi_{1}(t-1 ; q)-\phi_{1}(t ; q)\right]
\end{aligned}
$$


Now using Equation (29) the recursive relation of Example 4.1 can be obtained as

$$
\begin{aligned}
v_{1, m}(t)= & \left(\chi_{m}+h_{1}\right) v_{1, m-1}(t)-h_{1} v_{1}(t-1)-h_{1}\left(1-\chi_{m}\right) \mathbf{N}^{-}\left[\frac{1}{s}\left(1-\mathrm{e}^{2}\right)\right] \\
& -h_{1} \mathbf{N}^{-}\left\{\frac{u}{s} \mathbf{N}^{+}\left[R_{1}\left(v_{2, m-1}(t)\right)\right]\right\} \\
v_{2, m}(t)= & \left(\chi_{m}+h_{2}\right) v_{2, m-1}(t)-h_{2}\left(1-\chi_{m}\right) \mathbf{N}^{-}\left[\frac{1}{2 s}\left(\mathrm{e}^{2}-1\right)\right] \\
& +h_{2} \mathbf{N}^{-}\left\{\frac{u}{s} \mathbf{N}^{+}\left[R_{2}\left(v_{1, m-1}\right)(t)\right]\right\}, \quad m \geq 1
\end{aligned}
$$

By choosing an initial approximations of $v_{1,0}(t)=1$ and $v_{2,0}(t)=\frac{1}{2}\left(\mathrm{e}^{2}-1\right)$ and using Equation (44) we obtained the following

$$
\begin{aligned}
v_{1,1}(t) & =-2 h_{1}\left(\mathrm{e}^{2}-1\right) t, \quad v_{2,1}(t)=h_{2}\left(\mathrm{e}^{2}-1\right) t \\
v_{1,2}(t) & =-2 h_{1} h_{2}\left(\mathrm{e}^{2}-1\right) t^{2}-2 h_{1}\left(\mathrm{e}^{2}-1\right) t \\
v_{2,2}(t) & =\left(h_{2}^{2}+2 h_{1} h_{2}+h_{2}\right)\left(\mathrm{e}^{2}-1\right) t \\
v_{1,3}(t)= & \left(h_{1} h_{2}^{2}-3 h_{1} h_{2}\right)\left(\mathrm{e}^{2}-1\right) t^{2}-2 h_{1}\left(\mathrm{e}^{2}-1\right) t \\
v_{2,3}(t)= & -\frac{2}{3} h_{1} h_{2}\left(\mathrm{e}^{2}-1\right)^{2} t^{3}-\left(2 h_{1} h_{2}^{2}+h_{1} h_{2}\right)\left(\mathrm{e}^{2}-1\right)^{2} t^{2} \\
& +\left(h_{2}^{3}+2 h_{2}^{2}+h_{2}\right)\left(\mathrm{e}^{2}-1\right) t
\end{aligned}
$$

Following the same process remaining terms of $v_{i, m}(t)$ for $m \geq 3$ can be obtained. Putting $h_{1}=\frac{1}{3\left(\mathrm{e}^{2}-1\right)}$ and $h_{2}=-1$ in Equation (45), then the fifth order approximation of Example 4.1 is given as

$$
\begin{aligned}
v_{1}(t)= & 1-2 t+2 t^{2}-\frac{4}{3} t^{3}+\frac{2}{3} t^{4}-\frac{4}{15} t^{5}+\frac{4}{45} t^{6}+\cdots \\
v_{2}(t)= & \frac{1}{2}\left(\mathrm{e}^{2}-1\right)-\left(\mathrm{e}^{2}-1\right) t+\left(\mathrm{e}^{2}-1\right) t^{2}-\frac{2}{3}\left(\mathrm{e}^{2}-1\right) t^{3} \\
& +\frac{1}{3}\left(\mathrm{e}^{2}-1\right) t^{4}-\frac{2}{15}\left(\mathrm{e}^{2}-1\right) t^{5}+\frac{2}{45}\left(\mathrm{e}^{2}-1\right) t^{6}+\cdots
\end{aligned}
$$

The series solutions in Equation (46) converged to exact solutions $v_{1}(t)=\mathrm{e}^{-2 t}, v_{2}(t)=\frac{1}{2}\left(\mathrm{e}^{-2(t-1)}-\mathrm{e}^{-2 t}\right)$ of Equation (41).

Therefore, the fifth order approximated series of the derived algorithm in Equation (29) was successfully generates the closed form solution of Example 4.1 with minimum error as shown in Figure 1 While in most applications only numerical approximations was obtained. For instance, in [10] the numerical approximation of this problem was computed using Implicit Block method with the maximum absolute error of 1.47320 when the tolerance was $1 \times 10^{-10}$ in a total number of 25 steps.

Example 4.2 [12] Next we consider a third-order nonlinear system of NDDEs with both proportional and constant delays 


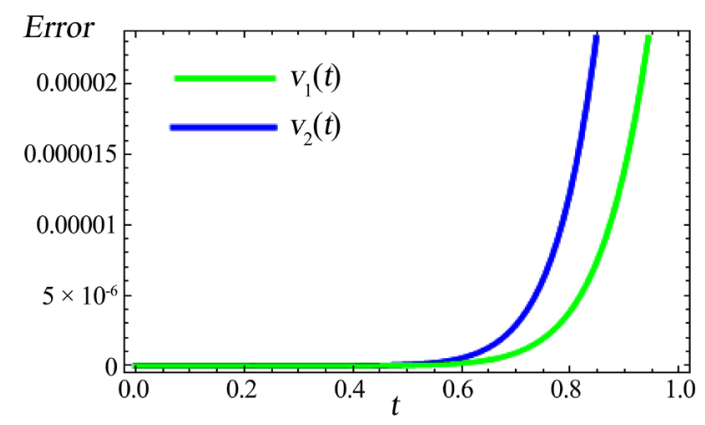

Figure 1. The behaviour of maximum absolute errors between the exact solution and fifth-order approximation of Example 4.1.

$$
\begin{aligned}
& v_{1}^{\prime \prime \prime}(t)=v_{1}^{\prime \prime \prime}(t-2) v_{1}\left(\frac{t}{3}\right)+\left(v_{1}(t)\right)^{\frac{2}{3}}+2 t+\mathrm{e}^{-t} \\
& v_{2}^{\prime \prime \prime}(t)=\frac{1}{2} v_{2}^{\prime \prime \prime}\left(\frac{t}{2}\right)+v_{2}^{\prime}(t-1) v_{1}\left(\frac{t}{3}\right), \quad t \geq 1
\end{aligned}
$$

with initial functions

$$
\phi_{1}(t)=\mathrm{e}^{t}, \quad \phi_{2}(t)=t^{2}, \quad t \in[-2,0]
$$

and the given initial conditions

$$
\begin{array}{ll}
v_{1}(0)=1, & v_{1}^{\prime}(0)=1, \quad v_{1}^{\prime \prime}(0)=1 \\
v_{2}(0)=0, & v_{2}^{\prime}(0)=0, \quad v_{2}^{\prime \prime}(0)=2
\end{array}
$$

Take the natural transform to both sides of Equation (47) and simplify further using Equation (11) to get

$$
\begin{aligned}
& \mathbf{N}^{+}\left[v_{1}(t)\right]-\left[\frac{1}{s}+\frac{u}{s^{2}}+\frac{u^{2}}{s^{3}}\right]-\frac{u^{3}}{s^{3}} \mathbf{N}^{+}\left[\mathrm{e}^{t-2} v_{1}\left(\frac{t}{2}\right)+\left(v_{1}(t)\right)^{\frac{2}{3}}+2 t+\mathrm{e}^{-t}\right]=0 \\
& \mathbf{N}^{+}\left[v_{2}(t)-8 v_{2}\left(\frac{t}{2}\right)\right]-\frac{u^{3}}{s^{2}} \mathbf{N}^{+}\left[2(1-t) v_{1}\left(\frac{t}{3}\right)\right]=0
\end{aligned}
$$

From Equation (48) define a non-linear operator

$$
\begin{aligned}
N\left[\phi_{1}(t ; q)\right]= & \mathbf{N}^{+}\left[\phi_{1}(t ; q)\right]-\left[\frac{1}{s}+\frac{u}{s^{2}}+\frac{u^{2}}{s^{3}}\right] \\
& -\frac{u^{3}}{s^{3}} \mathbf{N}^{+}\left[\mathrm{e}^{t-2} \phi_{1}\left(\frac{t}{2} ; q\right)+\left(\phi_{1}(t ; q)\right)^{\frac{2}{3}}+2 t+\mathrm{e}^{-t}\right] \\
N\left[\phi_{2}(t ; q)\right]= & \mathbf{N}^{+}\left[\phi_{2}(t ; q)-8 \phi_{2}\left(\frac{t}{2} ; q\right)\right]-\frac{u^{3}}{s^{2}} \mathbf{N}^{+}\left[2(1-t) \phi_{1}\left(\frac{t}{3} ; q\right)\right]=0
\end{aligned}
$$

Now using Equation (29) the recursive relation of Example 4.2 can be obtained as

$$
\begin{aligned}
v_{1, m}(t)= & \left(\chi_{m}+h_{1}\right) v_{1, m-1}-h_{1}\left(1-\chi_{m}\right) \mathbf{N}^{-}\left[\frac{1}{s}+\frac{u}{s^{2}}+\frac{u^{2}}{s^{3}}\right] \\
& -h_{1} \mathbf{N}^{-}\left\{\frac{u^{3}}{s^{3}} \mathbf{N}^{+}\left[R_{1}\left(v_{1, m-1}(t)\right)+H_{1, m-1}\left(v_{\lambda_{1}}, \cdots, v_{\lambda_{N}}\right)+g_{1}(t)\right]\right\} \\
v_{2, m}(t)= & \left(\chi_{m}+h_{2}\right) v_{2, m-1}(t)-4 h_{2} v_{m-1}\left(\frac{t}{2}\right)-h_{2} \mathbf{N}^{-}\left\{\frac{u^{3}}{s^{3}} \mathbf{N}^{+}\left[R_{2}\left(v_{2, m-1}\right)(t)\right]\right\}
\end{aligned}
$$


By choosing an initial approximations of $v_{1,0}(t)=1+t+\frac{t^{2}}{2 !}+\frac{t^{3}}{3 !}$ and $v_{2,0}(t)=t^{2}$ and using Equation (50) we obtained the following

$$
\begin{aligned}
v_{1,1}(t)= & -\left(\frac{1+\mathrm{e}^{-2}}{6}\right) h_{1} t^{3}-\left(\frac{5+4 \mathrm{e}^{-2}}{72}\right) h_{1} t^{4}-\left(\frac{16 \mathrm{e}^{-2}-5}{1080}\right) h_{1} t^{5} \\
& +\left(\frac{1098 \mathrm{e}^{-2}+506}{1145}\right) h_{1} t^{6} \\
v_{2,1}(t)= & \frac{1}{3} h_{2} t^{3}-\frac{1}{27} h_{2} t^{4}-\frac{1}{108} h_{2} t^{5}+\cdots
\end{aligned}
$$

By putting $h_{1}=-1$ and $h_{2}=-2$ in the series Equation (51) we obtained the approximate solution of Example 4.2 as

$$
\begin{aligned}
v_{1}(t)= & 1+t+\frac{t^{2}}{2}+\left(\frac{1+\mathrm{e}^{-2}}{6}\right) t^{3}+\left(\frac{5+4 \mathrm{e}^{-2}}{72}\right) t^{4}+\left(\frac{16 \mathrm{e}^{-2}-5}{1080}\right) t^{5} \\
& -\left(\frac{1098 \mathrm{e}^{-2}+506}{1145}\right) t^{6} \\
v_{2}(t)= & t^{2}-\frac{2}{3} t^{3}+\frac{2}{27} t^{4}+\frac{1}{54} t^{5}+\cdots
\end{aligned}
$$

Using only one iteration of the derived algorithm (first order) in Equation (29) a good approximation of Example 4.2 was successfully obtained. Since this problem has no exact solution therefore, Figure 2 shows the comparison between approximate solution obtained by the proposed technique, Matlab Package DDENSD and the result obtained by Rebenda and Smarda [12] using an algorithm based on the combination of the method of steps and differential transform method (DT).

Threrefore, from Figure 2 we can observed that there is a good correspondence between the first order approximate solution of the proposed tecnique with that of Matlab Package DDENSD and DT. Hence, this shows that the presented method provides reliable results and reduces the computation size as compared with the previous techniques.

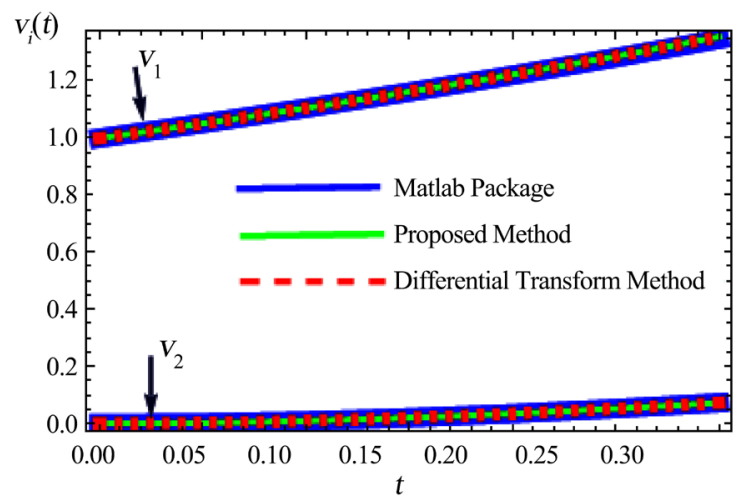

Figure 2. Comparison of solutions obtained by Matlab Package, Proposed Method and Differential Transform Method of Example 4.2. 


\section{Conclusion}

This paper presents an efficient analytical approach suitable for solving linear and nonlinear systems of NDDEs with proportional and constant delays via HAM and natural transform. The presented algorithm adjusted the He's polynomial in order to ease the computational difficulties of both proportional and constant delays. Another advantage of this research is that a new algorithm is constructed in Equation (25) which reduces the computational work as compared to other methods, produces a much faster convergent approximate solution and handles more complicated problems (as in the case of second example) in applications than other analytical methods. Therefore, the presented approach is efficient and reliable in solving different form of linear and nonlinear systems of NDDEs which can be also applied to solve various types of linear and nonlinear problems.

\section{Acknowledgements}

The authors like express their gratitude for financial support from Transdisciplinary Research Grant Scheme (TRGS) under the research programme of Characterisation of Spatio-temporal Marine Microalgae Ecological Impact Using Multi-Sensor Over Malaysia Waters for the specific sub-project of Mathematical Modelling of Harmful Algal Blooms (HABs) in Malaysia Waters (R.J130000.7809.4L854) funded by the Ministry of Education, Malaysia. Authors also like to appreciate the Universiti Teknologi Malaysia for providing all the necessary support and facilities toward the success of this research.

\section{Conflicts of Interest}

The authors declare no conflicts of interest regarding the publication of this paper.

\section{References}

[1] Alomari, A.K., Noorani, Mohd Salmi, M.D. and Nazar, R. (2009) Solution of Delay Differential Equation by Means of Homotopy Analysis Method. Acta Applicandae Mathematicae, 108, 395. https://doi.org/10.1007/s10440-008-9318-Z

[2] Batzel, J. and Tran, H.T. (2000) Stability of the Human Respiratory Control System I. Analysis of a Two-Dimensional Delay State-Space Model. Journal of Mathematical Biology, 41, 45-79. https://doi.org/10.1007/s002850000044

[3] Liu, L.P. and Kalmár-Nagy, T. (2010) High-Dimensional Harmonic Balance Analysis for Second-Order Delay-Differential Equations. Journal of Vibration and Control, 16, 1189-1208. https://doi.org/10.1177/1077546309341134

[4] Bellen, A. and Zennaro, M. (2013) Numerical Methods for Delay Differential Equations. Oxford University Press, Oxford.

[5] Rihan, F.A., et al. (2014) A Time Delay Model of Tumour-Immune System Interactions: Global Dynamics, Parameter Estimation, Sensitivity Analysis. Applied Mathematics and Computation, 232, 606-623.

https://doi.org/10.1016/j.amc.2014.01.111

[6] Rihan, F.A., Azamov, A.A. and Al-Sakaji, J.H. (2018) An Inverse Problem for Delay 
Differential Equations: Parameter Estimation, Nonlinearity, Sensitivity. Applied Mathematics, 12, 63-74. https://doi.org/10.18576/amis/120106

[7] Blanco-Cocom, L., Estrella, G.A. and Avila-Vales, E. (2012) Solving Delay Differential Systems with History Functions by the Adomian Decomposition Method. Applied Mathematics and Computation, 37, 5994-6011.

https://doi.org/10.1016/j.amc.2011.11.082

[8] Bellour, A. and Bousselsal, M. (2014) Numerical Solution of Delay Integro-Differential Equations by Using Taylor Collocation Method. Mathematical Methods in the Applied Sciences, 37, 1491-1506. https://doi.org/10.1002/mma.2910

[9] Duarte, J., Januario, C. and Martins, N. (2016) Analytical Solutions of an Economic Model by the Homotopy Analysis Method. Applied Mathematical Sciences, 10, 2483-2490. https://doi.org/10.12988/ams.2016.66188

[10] Ishak, F. and Ramli, M. S.B. (2015) Implicit Block Method for Solving Neutral Delay Differential Equations. AIP Conference Proceedings, 1682, Article ID: 020054. https://doi.org/10.1063/1.4932463

[11] Rebenda, J., Šmarda, Z. and Khan, Y. (2015) A Taylor Method Approach for Solving of Nonlinear Systems of Functional Differential Equations with Delay.

[12] Rebenda, J. and Šmarda, Z. (2019) Numerical Algorithm for Nonlinear Delayed Differential Systems of nth Order. Advances in Difference Equations, 2019, Article No. 26. https://doi.org/10.1186/s13662-019-1961-3

[13] Barde, A. and Maan, N. (2019) Efficient Analytical Approach for Nonlinear System of Delay Differential Equations. Computer Science, 14, 693-712.

[14] Liao, S.J. (1992) The Proposed Homotopy Analysis Technique for the Solution of Nonlinear Problems. Ph.D. Thesis, Shanghai Jiao Tong University, Shanghai.

[15] Liao, S.J. (2003) Beyond Perturbation: Introduction to the Homotopy Analysis Method. Chapman and Hall/CRC, New York. https://doi.org/10.1201/9780203491164

[16] Liao, S.J. and Cheung, F. (2003) Homotopy Analysis of Nonlinear Progressive Waves in Deep Water. Journal of Engineering Mathematics, 45, 105-116.

[17] Liao, S.J., Su, J. and Chwang, T.A. (2006) Series Solutions for a Nonlinear Model of Combined Convective and Radiative Cooling of a Spherical Body. International Journal of Heat and Mass Transfer, 49, 2437-2445. https://doi.org/10.1016/j.ijheatmasstransfer.2006.01.030

[18] Odibat, Z., Momani, S. and Xu, H. (2010) A Reliable Algorithm of Homotopy Analysis Method for Solving Nonlinear Fractional Differential Equation. Applied Mathematical Modelling, 34, 593-600. https://doi.org/10.1016/j.apm.2009.06.025

[19] Khan, Z.H. and Khan, W. (2008) N-Transform-Properties and Applications. NUST Journal of Engineering Sciences, 1, 127-133.

[20] Belgacem, F.B.M. and Silambarasan, R. (2012) Maxwell's Equations Solutions by Means of the Natural Transform. International Journal of Mathematics in Engineering, Science and Aerospace, 3, 313-323. https://doi.org/10.1063/1.4765477

[21] Loonker, D. and Banerji, P.K. (2014) Natural Transform and Solution of Integral Equations for Distribution Spaces. American Journal of Mathematics and Sciences, 3, 65-72.

[22] Al-Omari, S.K.Q. (2017) On q-Analogues of the Natural Transform of Certain q-Bessel Functions and Some Application. Filomat, 31, 2587-2598. https://doi.org/10.2298/FIL1709587A

[23] Belgacem, F.B.M. and Silambarasan, R. (2012) Theory of Natural Transform. Mathematics in Engineering, Science and Aerospace Journal, 3, 99-124. 
https://doi.org/10.1063/1.4765477

[24] Belgacem, S.R. (2012) Advances in the Natural Transform. AIP Conference Proceedings, 1493, 106. https://doi.org/10.1063/1.4765477 